\title{
On intersection of primary subgroups of odd order in finite almost simple groups
}

\author{
Viktor I. Zenkov \\ Ural Federal University, \\ Institute of Mathematics and Mechanics UB RAS, \\ V1I9Z52@mail.ru \\ Yakov N. Nuzhin \\ Siberian Federal University, \\ nuzhin2008@rambler.ru
}

Keywords: Finite group, almost simple group, primary subgroup.

Abstract

The question of the determination of subgroups $A$ and $B$ such that $A \cap B^{g} \neq 1$ for any $g \in G$ for a finite almost simple group $G$ and its primary subgroups $A$ and $B$ of odd order set up. We prove that there exist four possibilities for ordered pair $(A, B)$ only.

\section{Introduction}

Let $G$ be a finite group and $A, B$ be its subgroups. By the definition, $M$ is the set of subgroups which are minimal by inclusion among all subgroups of type $A \cap B^{g}, g \in G$, and $m$ consists of those elements of the set $M$ the order of which is minimal. $\operatorname{Set}_{\operatorname{Min}_{G}}(A, B)=\langle M\rangle$, and $\min _{G}(A, B)=$ $\langle m\rangle$. Evidently, $\operatorname{Min}_{G}(A, B) \geq \min _{G}(A, B)$ and the following three conditions are equivalent:

a) $A \cap B^{g} \neq 1$ for any $g \in G$;

б) $\operatorname{Min}_{G}(A, B) \neq 1$;

в) $\min _{G}(A, B) \neq 1$.

The second author showed that $\operatorname{Min}_{G}(A, B) \leq F(G)$ for any pair of abelian subgroups $A$ and $B$ of $G[4$, Theorem 1], where $F(G)$ is the Fitting subgroup of $G$ (the greatest normal nilpotent subgroup of $G$ ). On the other hand, V. I. Zenkov and V. D. Mazurov proved that $\operatorname{Min}_{G}(A, B)=1$ for any pair of primary subgroups $A$ and $B$ of a simple nonabelian group $G$ [3, Theorem 1]. But also in almost simple group $G \simeq \operatorname{Aut}\left(L_{2}(7)\right)$ we have $\operatorname{Min}_{G}(S, S)=\min _{G}(S, S)=S$ for a Sylow 2-subgroup $S$ of $G$. Moreover it is proved in [5] that for a group $G$ with socle $L_{2}(q), q>3$, if subgroups $A$ and $B$ are primary, then the inequality $\operatorname{Min}_{G}(A, B) \neq 1$ is valid only for $q=9$ and for the Mersenne prime $q=2^{n}-1$; in these cases subgroups $A$ and $B$ are 2-groups.

In the present article we consider the case when $A$ and $B$ are primary subgroups of odd order in a finite almost simple group $G$. Our main result is the following theorem.

Theorem 1. Let $G$ be a finite almost simple group and $A, B$ be its primary subgroups of odd order. Then the following are equivalent.

(1) $\operatorname{Min}_{G}(A, B) \neq 1$.

(2) $G$ contains a normal subgroup of index 1 or 2 , which is isomorphic to $D_{4}(3) \lambda Z_{3}$, and $(A, B) \in$ $\left\{S, S_{0}\right\}^{2}$. Where $S$ is a Sylow 3-subgroup of $G, S_{0}=O_{3}\left(N_{G}(P)\right)$ and $P$ is a minimal proper parabolic subgroup of the group $D_{4}(3)$ corresponding to the central node of the Coxeter graph of type $D_{4}$. Moreover $S_{0}=\min _{G}(S, S)$.

In section 2 we establish some necessary for the proof of theorem 1 properties of subgroups of Chevalley groups, which in the opinion of the authors and are of independent interest. 


\section{Notations and preliminary results}

A finite group $G$ is called almost simple, if $\operatorname{Inn}(K) \leq G \leq \operatorname{Aut}(K)$, where $K$ is a finite simple nonabelian group, and $\operatorname{Inn}(K)$ and $\operatorname{Aut}(K)$ are groups of inner and respectively all automorphisms of a group $K$. In this article the following shortcuts and notations are used:

$g^{h}=h^{-1} g h$ for elements $g$ and $h$ of a group $G$;

$A^{B}=\left\{a^{b} \mid a \in A, b \in B\right\}$ for subsets $A$ and $B$ of a group $G$;

$A \leq G$ denotes that $A$ is a subgroup of a group $G$;

$\langle M\rangle$ is the group generated by a set $M$;

$N_{G}(A)$ is the normalizer of a $\operatorname{subgroup} A$ in a group $G$;

$O_{p}(G)$ is the maximal normal $p$-subgroup of a finite group $G$;

$A \lambda B$ is the semidirect product of groups $A$ and $B$ with a normal subgroup $A$.

Actually, the proof of the implication $(1) \Rightarrow(2)$ of Theorem 1 is reduced to the analysis of situation in the group $D_{4}(3) \lambda Z_{3}$ by the usage of the following result of the second author.

Lemma 1. [5, Theorem $\mathrm{B}(2 \mathrm{a})]$ Let $G$ be a finite almost simple group, $p$ be an odd prime and $S$ be a Sylow p-subgroup of $G$. If $S \cap S^{g} \neq 1$ for any element $g$ of $G$, then $p=3$ and $G$ contains a normal subgroup of index 1 or 2, which is isomorphic to $D_{4}(3) \lambda Z_{3}$.

We will also need the following two technical lemmas, which will be use in the sequel.

Lemma 2. [5, Lemma 3.1] Let $G$ be a finite group and $M_{1}$ be a subgroup of $G$. Let $P_{1}$ be a Sylow p-subgroup of $M_{1}$ such that $P_{1} \cap P_{1}^{k}=O_{p}\left(M_{1}\right)$ for some $k \in M$, and $M_{2}$ be a conjugate with $M_{1}$ subgroup of $G$. Then there exists Sylow $p$-subgroup $P_{2}$ of $M_{2}$ such that $P_{1} \cap P_{2} \leq O_{p}\left(M_{1}\right) \cap O_{p}\left(M_{2}\right)$.

Lemma 3. Let $A, B, S$ be subgroups of a finite group $G$ such that $\operatorname{Min}_{G}(A, B) \neq 1, A \leq S$ and $A \cap B^{h}=S \cap S^{h}=T$ for some element $h \in G$ and some cyclic subgroup $T$ of prime order. Then $T^{S} \leq A$.

Proof. By the lemma conditions we have $T^{s}=S \cap S^{h s} \geq A \cap B^{h s} \neq 1$ for any $s \in S$. Therefore taking into account that $T$ is a cyclic subgroup of prime order, we deduce the inclusion $T^{s} \leq A$ for any $s \in S$, that is $T^{S} \leq A$. The lemma is proved.

\section{Some properties of intersections of Sylow $p$-subgroups of Chevalley groups over a finite field of characteristic $p$}

Further $\Phi$ is a reduced indecomposable root system, $\Pi=\left\{r_{1}, \ldots, r_{l}\right\}$ is its set of fundamental roots, $\Phi^{+}$is a positive root system respect to $\Pi$, and also $\Phi^{-}=-\Phi^{+}$. We always suggest, that $r_{1}$ is a short root and sum $r_{i}+r_{j}, i \leq j$, is a root if and only if:
$(i, j)=(l-3, l)$ or $(i, i+1), 1 \leq i \leq l-2$, if $\Phi=E_{l}$;
$(i, j)=(1,3)$ or $(i, i+1), 2 \leq i \leq l-1$, if $\Phi=D_{l}$;
$(i, j)=(i, i+1)$ in all other cases.

We will need the following strengthening the Lemma 3.6.2 from the book R. Carter [2, p. 50].

Lemma 4. Let a fundamental root $r_{i_{1}}$ be a part with nonzero coefficient of the expression of a root $r \in \Phi^{+}$as an integral combination of fundamental roots with non-negative coefficients. Then $r$ can be expressed as a sum of fundamental roots

$$
r=r_{i_{1}}+r_{i_{2}}+\cdots+r_{i_{k}}
$$

in such a way that $r_{i_{1}}+r_{i_{2}}+\cdots+r_{i_{s}}$ is a root for all $s \leq k$.

Proof. Let

$$
r=c_{1} r_{1}+\cdots+c_{l} r_{l}
$$


be the expression of a root $r \in \Phi^{+}$as an integral combination of fundamental roots with nonnegative coefficients. Obviously, that we have two following cases only: 1) $c_{i} \leq 1$; 2) at least one of numbers $c_{i}$ is more than one.

For any subgraph of the Coxeter graph of type $\Phi$, whose vertices are labeled by fundamental roots $r_{j_{1}}, \ldots, r_{j_{m}}$, the sum $r_{j_{1}}+\cdots+r_{j_{m}}$ if and only if is a root when this subgraph is connected. Therefore in the first case the lemma is true. The second case is reduced by induction to the first one by virtue of the following assertion.

A) If $c_{i}>1$ for some $i$, then there exists $j$ such that $c_{j}>1$ and the difference $r-r_{j}$ is a root.

Assertion A) can be directly verified for every root system.

For the root system of type $A_{l}$ the first case is possible only.

For the root system of type $B_{l}$ in the case 2) we have $r=2 r_{1}+\cdots+2 r_{s}+r_{s+1}+\cdots+r_{t}$, where $1 \leq s<t \leq l$. Here the one variant $j=s$ is possible only, in order that the difference $r-r_{j}$ is a root.

For the root system of type $C_{l}$ in the case 2) we have $r=r_{t}+\cdots+r_{s-1}+2 r_{s}+\cdots+2 r_{l-1}+r_{l}$, where $1 \leq t \leq s \leq l-1$ (for $s=1$ we set $r=2 r_{1}+\cdots+2 r_{l-1}+r_{l}$ ). Here also one variant $j=s$ is possible only.

For the root system of type $D_{l}$ in the case 2$)$ we have $r=r_{1}+r_{2}+2 r_{3}+\cdots+2 r_{s}+r_{s+1}+\cdots+r_{t}$, where $3 \leq s<t \leq l$. Here also one variant $j=s$ is possible only.

In the Tables V-VIII from [1] for the exceptional types $E_{l}$ and $F_{4}$ all positive roots, which have even one of numbers $c_{j}$ more than one are listed. Using these tables, it is not difficult to check the validity of the assertion $\mathrm{A}$ ) for the types $E_{l}$ and $F_{4}$. Note that even here for some roots the parameter $j$ is not uniquely defined.

For type $G_{2}$ the conclusion A) correctness is easily checked and in this case the parameter $j$ is defined uniquely.

Hence, the assertion A), along with lemma is proved.

Further $\Phi(q)$ is an adjoint Chevalley group of type $\Phi$ of rank $l$ over the finite field $\mathbb{F}_{q}$ of the order $q=p^{n}$, where $p$ is a prime. The group $\Phi(q)$ is generated by the root subgroups

$$
X_{r}=\left\langle x_{r}(t) \mid t \in K\right\rangle, r \in \Phi,
$$

where $x_{r}(t)$ is the corresponding root element in the group $\Phi(q)$. We will need the following natural subgroups of the group $\Phi(q)$ :

unipotent subgroups

$$
\begin{aligned}
& U=\left\langle X_{r} \mid r \in \Phi^{+}\right\rangle, \\
& V=\left\langle X_{r} \mid r \in \Phi^{-}\right\rangle,
\end{aligned}
$$

monomial subgroup

$$
N=\left\langle n_{r}(t) \mid r \in \Phi, t \in \mathbb{F}_{q}^{*}\right\rangle,
$$

diagonal subgroup

$$
H=\left\langle h_{r}(t) \mid r \in \Phi, t \in \mathbb{F}_{q}^{*}\right\rangle
$$

and Borel subgroup

$$
B=U H \text {. }
$$

Here, $\mathbb{F}_{q}^{*}$ is the multiplicative subgroup of the field $\mathbb{F}_{q}$ and

$$
\begin{gathered}
n_{r}(t)=x_{r}(t) x_{-r}\left(-t^{-1}\right) x_{r}(t), \\
h_{r}(t)=n_{r}(t) n_{r}(-1) .
\end{gathered}
$$

We set also

$$
I=\{1,2, \ldots, l\} .
$$

Overgroups of the Borel subgroup $B$ and conjugate with them are called parabolic. Due to familiar result of J.Tits, parabolic subgroups containing subgroup $B$ are

$$
P_{J}=\left\langle B, n_{r_{j}}(1) \mid j \in J\right\rangle,
$$


where $J \subseteq I$.

Lemma 5. Fix a monomial element $n_{0}$ with condition $U^{n_{0}}=V$ and a positive integer $i \in I$. Set $n=n_{0} n_{r_{i}}(1)$. Then $U \cap U^{n}=X_{r_{i}}$.

Proof. The root subgroups $X_{r_{i}}$ and $X_{-r_{i}}$ normalize the subgroup

$$
V_{r_{i}}=\left\langle X_{r} \mid r \in \Phi^{-} \backslash\left\{-r_{i}\right\}\right\rangle
$$

and $V=V_{r_{i}} X_{-r_{i}}\left[2\right.$, Lemma 8.1.1]. Therefore $U^{n}=V^{n_{r_{i}}}(1)=V_{r_{i}} X_{r_{i}}$. Clearly, that $U \cap V_{r_{i}} X_{r_{i}}=$ $X_{r_{i}}$. The lemma is proved.

For $l=1$ the root subgroup $X_{r_{i}}$ coincides with a Sylow $p$-subgroup of the group $\Phi(q)$ and in this case the element $n$ from Lemma 5 is diagonal.

Lemma 6. Let $P=P_{I \backslash\{i\}}$ be the parabolic maximal subgroup of the group $\Phi(q)$ of type $A_{l}$, $D_{l}$ or $E_{l}$ of rank $l \geq 2$ and the monomial element $n$ as in Lemma 5. Then $U \cap U^{n}=X_{r_{i}}$ and $\left\langle X_{r_{i}}^{U}\right\rangle=O_{p}(P)$.

Proof. For Chevalley groups $\Phi(q)$ of any type, the equality

$$
O_{p}(P)=\left\langle X_{r} \mid r=c_{k} r_{k}+\cdots+c_{i} r_{i}+\cdots+c_{m} r_{m}, 1 \leq k \leq i \leq m \leq l, c_{j} \geq 1\right\rangle .
$$

holds [2, Theorem 8.5.2]. For types $A_{l}, D_{l}$ and $E_{l}$, all structure constants of Chevalley's commutator formula are equal to 1 . Hence, using Lemma 4, we can obtain the equality $\left\langle X_{r_{i}}^{U}\right\rangle=O_{p}(P)$. Really, let $X_{r} \in O_{p}(P)$. Then by Lemma 4 as $i=i_{1}$ for the root $r$ we have the following representation

$$
r=r_{i_{1}}+r_{i_{2}}+\cdots+r_{i_{k}}
$$

where the sum $r_{i_{1}}+r_{i_{2}}+\cdots+r_{i_{s}}$ is the root for all $s \leq k$. Therefore, we obtain inclusions

$$
\begin{aligned}
& {\left[X_{r_{i_{1}}}, X_{r_{i_{2}}}\right]=X_{r_{i_{1}}+r_{i_{2}}} \subset\left\langle X_{r_{i}}^{U}\right\rangle,} \\
& {\left[X_{r_{i_{1}}+r_{i_{2}}}, X_{r_{i_{3}}}\right]=X_{r_{i_{1}}+r_{i_{2}}+r_{i_{3}}} \subset\left\langle X_{r_{i}}^{U}\right\rangle,} \\
& {\left[X_{r_{i_{1}}+\cdots+r_{i_{k-1}}}, X_{r_{k}}\right]=X_{r_{i_{1}}+\cdots+r_{i_{k}}}=X_{r} \subset\left\langle X_{r_{i}}^{U}\right\rangle .}
\end{aligned}
$$

Hence, $\left\langle X_{r_{i}}^{U}\right\rangle=O_{p}(P)$. Lemma 5 gives the equality $U \cap U^{n}=X_{r_{i}}$. The lemma is proved.

Lemma 6 conclusion can not be adapted in general for each types $B_{l}, C_{l}, F_{4}$ and $G_{2}$. For example, the following result holds.

Lemma 7. Let $P=P_{I \backslash\{1\}}$ be the parabolic maximal subgroup of the group $\Phi(2)$ of type $B_{l}$, $l \geq 2$, over the field of two elements, where $r_{1}$ is short root. There is no a root subgroup $X_{r}$ such that $\left\langle X_{r}^{U}\right\rangle=O_{p}(P)$.

Proof. Due to the parabolic maximal subgroup $P$ draw, the following equality is valid

$$
O_{p}(P)=\left\langle X_{s} \mid s=c_{1} r_{1}+\cdots+c_{k} r_{k}, 1 \leq k \leq l, c_{j} \geq 1\right\rangle .
$$

It is trivial, that the equality $\left\langle X_{r}^{U}\right\rangle=O_{p}(P)$ is admissible only for $r=r_{1}$. Note that subgroup $\left\langle X_{r_{1}}^{U}\right\rangle$ contains the product $x_{r_{1}+r_{2}}(1) x_{2 r_{1}+r_{2}}(1)$, but individually the elements $x_{r_{1}+r_{2}}(1)$ and $x_{2 r_{1}+r_{2}}(1)$ do not belong to $\left\langle X_{r_{1}}^{U}\right\rangle$. The lemma is proved.

The Picture 1 depicts correspondence between nodes of the Coxeter graph and roots from the fundamental root system, which are associated with the Chevalley group of type $D_{4}$, is recognized. 


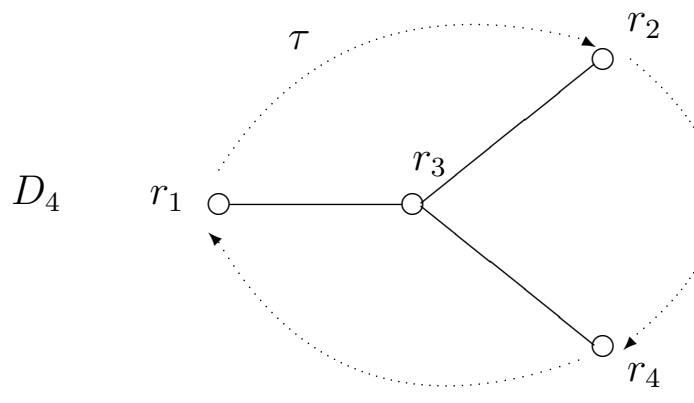

Pic. 1

Lemma 8. Let $G=D_{4}(3) \lambda\langle\tau\rangle$ and $S=U \lambda\langle\tau\rangle$, where $\tau$ is the graph automorphism of order 3 of the group $D_{4}(3)$ as on the picture 1 , and let the monomial element $n=n_{0} n_{r_{1}}(1) \in D_{4}(3)$ be as in Lemma 5. Then $S \cap S^{n}=X_{r_{1}}$, in particular, $U \cap U^{n}=X_{r_{1}}$.

Proof. In the Weyl group of type $D_{4}$ there exists an element $w_{0}$ such that $w_{0}(r)=-r$ for any root $r$. Moreover, $w_{0}$ coincides with cube $c^{3}$ of the Coxeter element

$$
c=w_{r_{3}} w_{r_{1}} w_{r_{2}} w_{r_{4}} .
$$

Hence in our case the element $n_{0}$ is the preimage of the element $w_{0}$ under the homomorphism of the monomial subgroup of the group $D_{4}(3)$ on the Weyl group of type $D_{4}$. Moreover, we can suggest, that

$$
n_{0}=\left(n_{r_{3}}(1) n_{r_{1}}(1) n_{r_{2}}(1) n_{r_{4}}(1)\right)^{3} .
$$

Since the graph automorphism $\tau$ centralizes the monomial element

$$
n_{r_{3}}(1) n_{r_{1}}(1) n_{r_{2}}(1) n_{r_{4}}(1)
$$

(see Picture 1 and [2, Proposition 12.2.3]), then

$$
\tau^{n}=\tau^{n_{r_{1}}(1)}=\tau n_{r_{2}}(-1) n_{r_{1}}(1) .
$$

It is clear that $\tau n_{r_{2}}(-1) n_{r_{1}}(1) \notin\langle\tau\rangle$. Therefore, and from the equality $U \cap U^{n}=X_{r_{1}}$, which is given by Lemma 5 , we obtain the equality $S \cap S^{n}=X_{r_{1}}$. The lemma is proved.

Lemma 9. Let $G, S$ and $\tau$ as Lemma 8. Set $S_{0}=U_{r_{3}} \lambda\langle\tau\rangle$, where $U_{r_{3}}=\left\langle X_{r} \mid r \in \Phi^{+} \backslash\left\{r_{3}\right\}\right\rangle$. Then $S_{0}=\min _{G}(S, S)$.

Proof. The subgroup $S$ is a Sylow 3 -subgroup of $G$. Therefore, by Lemma $1 S \cap S^{x} \neq 1$ for any $x \in G$, hence $\min _{G}(A, B)=\langle m\rangle \neq 1$ and by Lemma 5 the set $m$ consists of subgroups of order 3. Further, in the Coxeter graph of type $D_{4}$ the roots $r_{1}, r_{2}$ and $r_{4}$ coincide with symmetric nodes (see Picture 1), hence Lemma 8 is valid, if the root $r_{1}$ is exchanged with the root $r_{2}$ or $r_{4}$. Therefore by Lemma $6 O_{3}\left(P_{\{i\}}\right) \leq \min _{G}(S, S)$ for any $i=1,2,4$. Thus, Lemmas $1,5,6$ and 8 along with the equality

$$
\left\langle O_{3}\left(P_{I \backslash\{1\}}\right), O_{3}\left(P_{I \backslash\{2\}}\right), O_{3}\left(P_{I \backslash\{4\}}\right)\right\rangle=O_{3}\left(P_{\{3\}}\right)
$$

give the inclusion $S_{0} \leq \min _{G}(S, S)$.

Suppose $S_{0}<\min _{G}(S, S)$. Then there is exists an element (subgroup) $D$ of the set $m$ such that $D=S \cap S^{g} \not \leq S_{0}$ for some $g \in G$. Because of $\left|S \cap S^{g}\right|=3$ by Lemma 8, then $S_{0} \cap S_{0}^{g}=1$. Subgroups $S$ and $S_{0}$ satisfy the conditions of Lemma 2 as

$$
\begin{gathered}
G=D_{4}(3) \lambda\langle\tau\rangle, \\
M_{1}=N_{G}\left(P_{\{3\}}\right), \\
P_{1}=S,
\end{gathered}
$$




$$
\begin{gathered}
O_{3}\left(M_{1}\right)=S_{0}, \\
k=n_{r_{3}}(1) \\
O_{3}\left(M_{2}\right)=S_{0} .
\end{gathered}
$$

Thus by Lemma 2

$$
S \cap S^{x} \leq S_{0} \cap S_{0}^{g}=1
$$

for suitable $x \in G$. Hence, $S \cap S^{x}=1$. Contradiction with Lemma 1 . The lemma is proved.

\section{The proof of the Theorem 1}

Let a group $G$ satisfies the conditions of Theorem 1. Further, we use the notations of the preceding paragraph for subgroups and elements of the Chevalley group $D_{4}(3)$.

$(1) \Rightarrow(2)$ Let $\operatorname{Min}_{G}(A, B) \neq 1$. Since $A$ and $B$ are primary subgroups, then the condition $\operatorname{Min}_{G}(A, B) \neq 1$ implies that subgroups $A$ and $B$ are $p$-groups for an odd prime $p$. Hence, without loosing generality, we can suggest, that subgroups $A$ and $B$ lie in one fixed Sylow $p$-subgroup $S$ of the group $G$. Now again by the condition $\operatorname{Min}_{G}(A, B) \neq 1$ we get the inequality $S \cap S^{g} \neq 1$ for any element $g$ of $G$. Therefore by Lemma 1 we can suggest, that $p=3$ and the group $G$ contains the normal subgroup

$$
G_{0}=D_{4}(3) \lambda\langle\tau\rangle
$$

of index 1 or 2, where $\tau$ is a graph automorphism of order 3 of the group $D_{4}(3)$. We can suggest, that

$$
S=U \lambda\langle\tau\rangle .
$$

Let

$$
n_{0}=\left(n_{r_{3}}(1) n_{r_{1}}(1) n_{r_{2}}(1) n_{r_{4}}(1)\right)^{3} .
$$

Then (see the proof of Lemma 8)

$$
S \cap S^{n_{0}}=\langle\tau\rangle .
$$

Since

$$
\langle\tau\rangle=S \cap S^{n_{0}} \geq A \cap B^{n_{0}} \neq 1,
$$

then we obtain

$$
A \cap B^{n_{0}}=\langle\tau\rangle .
$$

By Lemma 8 there exists a monomial element $n \in D_{4}(3)$ such that

$$
S \cap S^{n}=X_{r_{1}} .
$$

Since

$$
X_{r_{1}}=S \cap S^{n} \geq A \cap B^{n} \neq 1,
$$

then

$$
A \cap B^{n_{0}}=X_{r_{1}} .
$$

Now by Lemma 3, we have

$$
X_{r_{1}}^{S} \leq A
$$

By Lemma 6,

$$
\left\langle X_{r_{1}}^{U}\right\rangle=O_{3}\left(P_{I \backslash\{1\}}\right) .
$$

As $\tau \in A$,

$$
\left\langle O_{3}\left(P_{I \backslash\{1\}}\right), O_{3}\left(P_{I \backslash\{1\}}^{\tau}\right), O_{3}\left(P_{I \backslash\{1\}}^{\tau^{2}}\right)\right\rangle=O_{3}\left(P_{\{3\}}\right) \leq A .
$$

Hence

$$
O_{3}\left(N_{G}\left(P_{\{3\}}\right)\right) \leq A \text {. }
$$

Suppose that

$$
S_{0}=O_{3}\left(N_{G}\left(P_{\{3\}}\right)\right) \text {. }
$$


Note, that $\left|S: S_{0}\right|=3$. Therefore, either $A=S_{0}$ or $A=S$. The condition $A \cap B^{g} \neq 1$ for any $g \in G$ is equivalent to the condition $B \cap A^{g^{-1}} \neq 1$ for any $g \in G$. Thus

$$
(A, B) \in\left\{S, S_{0}\right\}^{2} .
$$

It remains only to prove equality $S_{0}=\min _{G}(S, S)$. We have two cases only: a) $G=G_{0}$; b) $\left|G: G_{0}\right|=2$.

In case a), the equality $S_{0}=\min _{G}(S, S)$ is valid by Lemma 9 .

The case b) follows from a) and invariance of subgroup $S$ with respect to outer (graph) automorphisms of order 2 of the group $D_{4}(3)$.

$(1) \Leftarrow(2)$. It is clear that for pair $(S, S)$ the inequality $S \cap S^{g} \neq 1$ for any element $g \in G$ follows from recently found equality $S_{0}=\min _{G}(S, S)$.

As it is already known, that $S \cap S^{g} \neq 1$ for any $g \in G$ and $S \cap S^{n_{r_{3}}(1)}=S_{0}$, then by Lemma 2 (see the proof of Lemma 9) we have $S_{0} \cap S_{0}^{g} \neq 1$ and, moreover, $S_{0} \cap S^{g} \neq 1$ and $S \cap S_{0}^{g} \neq 1$ for any $g \in G$. This concludes the proof of our theorem.

The work of the first author is supported by RFBR (project 13-01-00469), by the Program of the Division of Mathematical Sciences of RAS (project 12-T-1-10003), by the Program of the Joint Investigations of RAS with SB RAS (project 12-C-1-1018) and with UB Belarussian National Academy of Sciences (project 12-C-1-1009) and by the Program of the State support of leading universities of the Russia (agreement no. 02.A03.210006 from 27.08.2013). The work of the second author is supported by RFBR (project 12-01-00968).

\section{References}

[1] N.Bourbaki. Groupes et algebres de Lie. VI-VI (Hermann, 1968).

[2] R.W.Carter. Simple groups of Lie type (Wiley and Sons, 1972).

[3] V.D.Mazurov, V.I.Zenkov, On intersections of Sylow subgroups in finite groups, Algebra $i$ logika., 35(1996), №4, 424-432.

[4] V.I.Zenkov, Intersections of Abelian subgroups in finite groups, Matemat. zametki., 56(1994), №2, 150-152.

[5] V.I.Zenkov, Intersection of nilpotent subgroups in finite groups, Fundamental. i prikladnaya matematika, 2(1996), №1, 1-92. 